\title{
The Stellar Black Hole
}

\section{Kenneth Dalton}

89/2 M.1 Th. Pongprasart, Bang Saphan, Thailand

Email:kxdalton@yahoo.com

How to cite this paper: Dalton, K. (2018) The Stellar Black Hole. Journal of High Energy Physics, Gravitation and Cosmology, 4, 651-654.

https://doi.org/10.4236/jhepgc.2018.44037

Received: June 27, 2018

Accepted: October 5, 2018

Published: October 8, 2018

Copyright $\odot 2018$ by author and Scientific Research Publishing Inc.

This work is licensed under the Creative

Commons Attribution International

License (CC BY 4.0).

http://creativecommons.org/licenses/by/4.0/

\begin{abstract}
A black hole model is proposed in which a neutron star is surrounded by a neutral gas of electrons and positrons. The gas is in a completely degenerate quantum state and does not radiate. The pressure and density in the gas are found to be much less than those in the neutron star. The radius of the black hole is far greater than the Schwarzschild radius.
\end{abstract}

\section{Keywords}

Black Hole Model, Neutron Stars, Degenerate Lepton Gas

\section{Introduction}

In the following model, a neutron star forms the core of a stellar black hole. The collapse of a massive star creates the neutron star. Electron-positron pairs, which are produced during the collapse, cannot escape the intense gravitational field of the neutron star. They settle into a degenerate quantum state surrounding the core.

The Fermi energy for a completely degenerate $(T=0)$ gas of $N / 2$ electrons is given by [1] [2]

$$
\epsilon_{F}=\left(\frac{3 \pi^{2}}{2}\right)^{2 / 3} \frac{\hbar^{2}}{2 m} n^{2 / 3}
$$

where $n$ is the total number density of leptons. If the gas is non-relativistic, the Fermi level must be less than $0.5 \mathrm{MeV}$. Formula (1) then limits the number density to about $3 \times 10^{30} \mathrm{~cm}^{-3}$, with the corresponding mass density $\rho=n m=3 \times 10^{3} \mathrm{~g} \cdot \mathrm{cm}^{-3}$. Therefore, the density of the lepton gas will be much less than that of the core $\left(\rho_{c}=5 \times 10^{14} \mathrm{~g} \cdot \mathrm{cm}^{-3}\right)$. Clearly, the gas envelope will have little direct impact on the core.

\section{The Black Hole Model}

Under the assumption of spherical symmetry, the gravitational field equation 
and the equation of hydrostatic equilibrium are given by

$$
\frac{1}{r^{2}} \frac{\mathrm{d}}{\mathrm{d} r}\left(r^{2} \frac{\mathrm{d} \psi}{\mathrm{d} r}\right)=4 \pi G \rho
$$

and

$$
\frac{\mathrm{d} P}{\mathrm{~d} r}=-\rho \frac{\mathrm{d} \psi}{\mathrm{d} r}
$$

If the lepton gas is assumed to be incompressible, then the density is constant, and Equation (2) gives

$$
\frac{\mathrm{d} \psi}{\mathrm{d} r}=\frac{4 \pi G}{3} \rho r+\frac{K}{r^{2}}
$$

At the surface of the neutron star, $\mathrm{d} \psi / \mathrm{d} r=G M_{c} / R_{c}^{2}$, where $M_{c}$ and $R_{c}$ are the mass and radius of the core. Substitute this into (4) to find

$$
K=G M_{c}\left(1-\frac{\rho}{\rho_{c}}\right)
$$

Since $\rho \ll \rho_{c}$, the constant of integration $K=G M_{c}$. Equations (3) and (4) then combine to give

$$
P=\frac{G M_{c}}{R_{c}} \rho\left(\frac{R_{c}}{r}-\frac{\rho}{2 \rho_{c}} \frac{r^{2}}{R_{c}^{2}}\right)+P^{\prime}
$$

At the outer surface of the gas, $r=R$ and $P=0$, so that the integration constant is

$$
P^{\prime}=-\frac{G M_{c}}{R_{c}} \rho\left(\frac{R_{c}}{R}-\frac{\rho}{2 \rho_{c}} \frac{R^{2}}{R_{c}^{2}}\right)
$$

This yields the pressure formula for the gas

$$
P=\frac{G M_{c}}{R_{c}} \rho\left\{R_{c}\left(\frac{1}{r}-\frac{1}{R}\right)+\frac{\rho}{2 \rho_{c}} \frac{1}{R_{c}^{2}}\left(R^{2}-r^{2}\right)\right\}\left(R_{c}<r<R\right)
$$

The maximum pressure occurs at the surface of the core $\left(r=R_{c}\right)$

$$
P_{\max }=\frac{G M_{c}}{R_{c}} \rho\left\{\left(1-\frac{R_{c}}{R}\right)+\frac{\rho}{2 \rho_{c}}\left(\frac{R^{2}}{R_{c}^{2}}-1\right)\right\}
$$

From the previous discussion regarding densities, it is clear that the radius of the gas envelope will be much greater than the core radius $R \gg R_{c}$, so that (9) reduces to

$$
P_{\max }=\frac{G M_{c}}{R_{c}} \rho\left(1+\frac{\rho}{2 \rho_{c}} \frac{R^{2}}{R_{c}^{2}}\right)
$$

Since $\rho \ll \rho_{c}$, it follows that $\rho R^{2} \ll 2 \rho_{c} R_{c}^{2}$ for any reasonable black hole mass. This yields the formula

$$
P_{\max }=\frac{G M_{c}}{R_{c}} \rho
$$

which will be used to determine the physical conditions near the core. 
The pressure in the electron-positron gas is given by

$$
P_{F}=\left(\frac{3 \pi^{2}}{2}\right)^{2 / 3} \frac{\hbar^{2}}{5 m} n^{5 / 3}
$$

Equate this to (11) and set $\rho=n m$ to obtain

$$
\left(\frac{3 \pi^{2}}{2}\right)^{2 / 3} \frac{\hbar^{2}}{5 m} n^{2 / 3}=\frac{G M_{c} m}{R_{c}}
$$

or

$$
n=5^{1 / 2} \frac{10 m^{3}}{3 \pi^{2} \hbar^{3}}\left(\frac{G M_{c}}{R_{c}}\right)^{3 / 2}
$$

A neutron star core, with $M_{c}=1.5 M_{\odot}$ and $R_{c}=1.1 \times 10^{6} \mathrm{~cm}$, yields the number density for the lepton gas

$$
n=1.2 \times 10^{30} \mathrm{~cm}^{-3}
$$

and the mass density

$$
\rho=m n=1.1 \times 10^{3} \mathrm{~g} \cdot \mathrm{cm}^{-3}
$$

Typical black hole dimensions are as follows: $M=5 M_{\odot}, R=1.1 \times 10^{10} \mathrm{~cm}$; $M=10 M_{\odot}, R=1.5 \times 10^{10} \mathrm{~cm} ; M=20 M_{\odot}, R=2.0 \times 10^{10} \mathrm{~cm}$. They are nearly the size of a normal star.

\section{Remarks}

Formula (14) shows that the density of the gas is determined by the intense gravitational field of the neutron star. It is little affected by the mass of the lepton gas itself. This results in a nearly common gas density for all stellar black holes. According to (11), the gas pressure near the core is $2 \times 10^{22} \mathrm{~Pa}$. As expected, this is much less than the pressure within the neutron star $\left(10^{34} \mathrm{~Pa}\right)$.

The very thick cloud of leptons is a conductor of electricity. It would shield the magnetic field of the neutron star. Moreover, the cloud would absorb much of the angular momentum of the parent star, thereby reducing the neutron star's rotation rate. In a more realistic treatment, the pressure and density would be related everywhere as in Formula (12), so that they both decrease with elevation. The resulting decrease in Fermi energy enables the upper layers of gas to interact with radiation coming from the core. During a collision with another black hole or neutron star, the lepton cloud could absorb a great deal of the electromagnetic radiation. Gravitational radiation would pass through the cloud unhindered.

Finally, it is worth emphasizing that the stellar black holes modeled here are those listed online [3]. They are not hypothetical. They are objects which have become paired with an observable star in a binary system. The astronomers have found that they have no magnetic field, they do not emit radiation, and they are always more massive than a neutron star. This model explains the experimental facts.

Note: The model treated here is incompressible. However, Formula (12) 
shows that the density of a degenerate gas varies with pressure. It is a polytrope, which satisfies the Lane-Emden equation [4] [5]. The standard Lane-Emden solution requires the initial condition $\mathrm{d} P / \mathrm{d} r=0$ at the center. This condition cannot be met in the present case, because of the neutron star's intense gravitational field near $r=R_{c}$ A new composite solution must be found, in order to improve upon the calculation given here.

\section{Conflicts of Interest}

The author declares no conflicts of interest regarding the publication of this paper.

\section{References}

[1] Landau, L. and Lifschitz, E. (1980) Statistical Physics Part 1. 3rd Edition, Pergamon, Section 57.

[2] Pathria, R. and Beale, P. (2011) Statistical Mechanics. 3rd Edition, Elsevier, Amsterdam, Chapter 8.

[3] Stellar Black Hole. https://en.m.wikipedia.org

[4] Chandrasekhar, S. (1958) An Introduction to the Study of Stellar Structure. Dover, Chapter IV.

[5] Horedt, G.P. (2004) Polytropes. Kluwer, Alphen aan den Rijn, Section 2.1. 